\title{
Nonlinear Pressure Wave Analysis by Concentrated Mass Model*
}

\author{
(1st Report, Suggestion and Validity Verification \\ of Analytic Model)
}

\author{
Satoshi ISHIKAWA ${ }^{* *}$, Takahiro KONDOU ${ }^{* * *}$ and Kenichiro MATSUZAKI ${ }^{* * *}$ \\ **Department of Intelligent Machinery and Systems, Kyushu University, \\ 744 Motooka, Nishi-ku, Fukuoka-shi, Fukuoka 819-0395, Japan \\ E-mail: ishikawa@mvib.mech.kyushu-u.ac.jp \\ ${ }^{* * * D e p a r t m e n t ~ o f ~ M e c h a n i c a l ~ E n g i n e e r i n g, ~ F a c u l t y ~ o f ~ E n g i n e e r i n g, ~ K y u s h u ~ U n i v e r s i t y, ~}$ \\ 744 Motooka, Nishi-ku, Fukuoka-shi, Fukuoka 819-0395, Japan
}

\begin{abstract}
A pressure wave propagating in a tube often changes to a shock wave because of the nonlinear effect of fluid. Analyzing this phenomenon by the finite difference method requires high computational cost. To lessen the computational cost, a concentrated mass model is proposed. This model consists of masses, connecting nonlinear springs, connecting dampers, and base support dampers. The characteristic of a connecting nonlinear spring is derived from the adiabatic change of fluid, and the equivalent mass and equivalent damping coefficient of the base support damper are derived from the equation of motion of fluid in a cylindrical tube. Pressure waves generated in a hydraulic oil tube, a sound tube and a plane-wave tube are analyzed numerically by the proposed model to confirm the validity of the model. All numerical computational results agree very well with the experimental results carried out by Okamura, Saenger and Kamakura. Especially, the numerical analysis reproduces the phenomena that a pressure wave with large amplitude propagating in a sound tube or in a plane tube changes to a shock wave. Therefore, it is concluded that the proposed model is valid for the numerical analysis of nonlinear pressure wave problem.
\end{abstract}

Keywords: Method of Vibration Analysis, Simulation, Modeling, Vibration Coupled with Fluid Motion, Nonlinear Vibration, Shock Wave, Nonlinear Wave, Viscous Flow

\section{Introduction}

A pressure wave propagating in a tube often changes to a shock wave because of the nonlinear effect of fluid. For example, in an engine exhaust pipe, the pressure wave becomes a shock wave, and a high-frequency noise appears ${ }^{(1),(2)}$. Similarly, in the tunnel of a high-speed train, a compression wave in front of the train changes to a shock wave as the wave propagates along the tunnel ${ }^{(3)}$.

When these shock waves occur, they often cause a serious noise problem, and so it is a very important issue to identify the characteristics of the nonlinear wave phenomena. Therefore, basic studies have been conducted. For example, a study of a shock wave occurring in a sound tube excited by a piston ${ }^{(4)-(6)}$, and an experiment of the phenomenon that a sinusoidal wave generated by a horn driver changes to a shock wave in a straight tube $^{(7)}$ have been carried out. Numerical analyses ${ }^{(2),(3),(6)}$ of these phenomena by the finite 
difference method were conducted. Because such analyses require high computational cost, an analysis method based on a convenient model is necessary.

Therefore, in the present paper, we examine the one-dimensional nonlinear pressure wave phenomena and propose a concentrated mass model. This model consists of masses, connecting nonlinear springs, connecting dampers, and base support dampers. The characteristic of connecting nonlinear springs is derived from the adiabatic change of fluid, and the connecting damper is derived from the normal stress of a fluid. Based on research by D'Souza ${ }^{(8)}$, the equivalent mass and equivalent damping coefficient of the base support damper are derived from the viscosity of fluid and velocity distribution. Then, pressure waves generated in a hydraulic oil tube $e^{(9)}$, in a sound tube ${ }^{(5)}$ and in a plane-wave tube ${ }^{(7)}$ are analyzed numerically by the proposed model to confirm the validity of the model.

\section{Concentrated Mass Model}

We consider the one-dimensional nonlinear pressure wave phenomenon in a cylindrical tube with a uniform cross-section. The fluid is modeled as a concentrated mass model which consists of masses, connecting nonlinear springs, connecting dampers, and base support dampers, as shown in Fig. 1, to analyze the pressure wave phenomenon. We neglect a steady flow. The fluid is divided into $N$-plex uniform elements such that the radius is $R$ and the cross-sectional area is $A$. The mass is concentrated on the nodal points. The mass in each nodal point and the length of each element in equilibrium state are uniform. The displacement in nodal point $i$ is $x_{i}$, the pressure in element $i$ is $p_{i}$, the variation of the pressure is $d p_{i}$, and the pressure in equilibrium state is $p_{0}$. The pressure in element $i$ is written as follows

$$
p_{i}=p_{0}+d p_{i} \text {. }
$$

\subsection{Nonlinear spring}

A nonlinear spring describes the relationship between pressure $p_{i}$ and volume $V_{i}$ in each element. If we regard the fluid as an ideal gas, and assume an adiabatic change, we obtain

$$
p_{i} V_{i}^{\gamma}=p_{0}(A l)^{\gamma},
$$

where $\gamma$ is the ratio of specific heats, and

$$
V_{i}=A\left(l+x_{i}-x_{i-1}\right) .
$$

Further, we assume $f_{i}^{k}$ is a variation of the nonlinear restoring force acting on the spring in element $i$, such that

$$
f_{i}^{k}=-A d p_{i} .
$$

Equations (1) (4) give

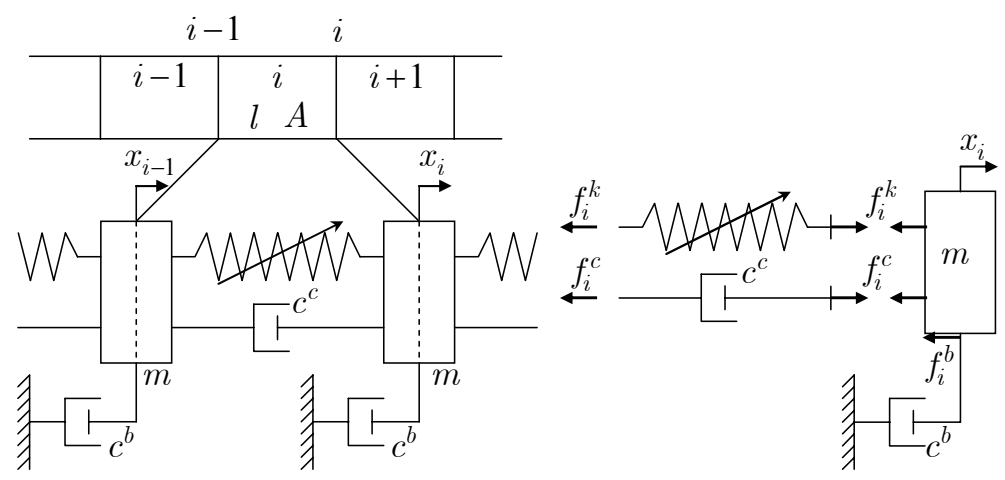

Fig. 1 Concentrated mass model 


$$
f_{i}^{k}=p_{0} A\left\{1-\frac{l^{\gamma}}{\left(l+x_{i}-x_{i-1}\right)^{\gamma}}\right\} .
$$

Assuming $f_{i}^{k L}$ is the linearized restoring force from Eq. (5), $f_{i}^{k L}$ is written as follows

$$
f_{i}^{k L}=\frac{p_{0} A \gamma\left(x_{i}-x_{i-1}\right)}{l} .
$$

And so, the linearized constant of spring $k^{L}$ is

$$
k^{L}=\frac{p_{0} A \gamma}{l} .
$$

If a bulk modulus $K$ is given, $k^{L}$ is represented as

$$
k^{L}=\frac{K A}{l} .
$$

\subsection{Mass and base support damper}

We regard the fluid flow in the element as an unsteady axisymmetric flow having a velocity distribution dependent on the viscosity when the nonlinear wave phenomena appear. In this section, we develop the expression of the mass $m$ and the damping coefficient of base support damper $c_{b}$ in the concentrated mass model shown in Fig. 1. The control volume is the shape of thin wall pipe with radius $r$, and thickness $d r$ in the shaded area represented by nodal point $i$ in Fig. 2. We define $\mu_{b}$ as the viscosity about the shear stress, $\rho_{0}$ as the density in equilibrium, and $u(r)$ as the velocity with radius $r$ in the control volume. If we assume pressure $p_{i}$ acts on the left side of the control volume, and $p_{i+1}$ acts on the right side, the equation of motion of the control volume is given by

$$
2 \pi r d r \rho_{0} l \frac{\partial u}{\partial t}=2 \pi r d r l \mu_{b}\left(\frac{\partial^{2} u}{\partial r^{2}}+\frac{1}{r} \frac{\partial u}{\partial r}\right)-2 \pi r d r\left(p_{i+1}-p_{i}\right) .
$$

Now, assuming that the variation of pressure $d p_{i}$ vibrates harmonically, the variation of pressure is defined by

$$
d p_{i}=d \tilde{p}_{i} e^{j \omega t},
$$

where $\omega$ is the angular frequency and $j$ is the imaginary unit. Substituting Eq. (10) into Eq. (9), we obtain

$$
\begin{aligned}
& 2 \pi r d r \rho_{0} l \frac{\partial u}{\partial t}=2 \pi r d r l \mu_{b}\left(\frac{\partial^{2} u}{\partial r^{2}}+\frac{1}{r} \frac{\partial u}{\partial r}\right)-2 \pi r d r \Delta \tilde{p}_{i} e^{j \omega t}, \\
& \Delta \tilde{p}_{i}=d \tilde{p}_{i+1}-d \tilde{p}_{i} .
\end{aligned}
$$

We develop the expression of the velocity $u(r)$ and the cross-sectional average velocity $\bar{u}$ by using a method similar to that by D'Souza ${ }^{(8)}$. First, we assume

$$
u=\left\{\phi(r)-\frac{\Delta \tilde{p}_{i}}{j \omega \rho_{0} l}\right\} e^{j \omega t} .
$$

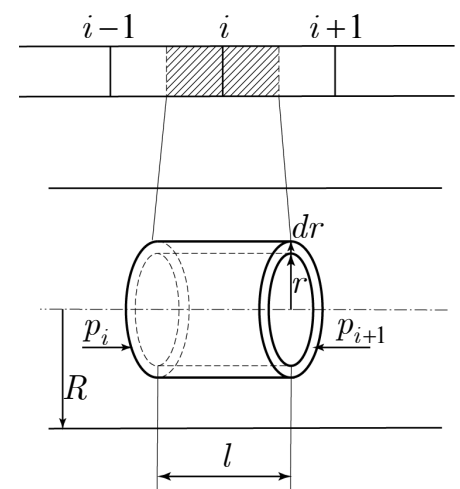

Fig. 2 Control Volume 
Substituting Eq. (12) into Eq. (11), we obtain

$$
\left(\frac{\partial^{2}}{\partial r^{2}}+\frac{1}{r} \frac{\partial}{\partial r}-\frac{j \omega}{\nu}\right) \phi=0
$$

where $\nu=\mu_{b} / \rho_{0}$. The solution of Eq. (13) is given by

$$
\phi=C J_{0}(j \lambda r), \quad \lambda=\sqrt{\frac{j \omega}{\nu}},
$$

where $J_{n}(z)$ is a Bessel function of the first kind and order $n$, and $C$ is a constant. Considering the boundary condition that satisfies $u(R)=0$ at the wall, we derive

$$
C=\frac{\Delta \tilde{p}_{i}}{j \omega \rho_{0} l} \frac{1}{J_{0}(j \lambda R)} .
$$

Equations (12), (14), (15) give

$$
u(r)=-\frac{\Delta \tilde{p}_{i}}{j \omega \rho_{0} l}\left\{1-\frac{J_{0}(j \lambda r)}{J_{0}(j \lambda R)}\right\} e^{j \omega t} .
$$

Using $u(r)$, the cross-sectional average velocity $\bar{u}$ is written as follows

$$
\bar{u}=\frac{1}{\pi R^{2}} \int_{0}^{R} 2 \pi r u d r=-\frac{\Delta \tilde{p}_{i}}{j \omega \rho_{0} l}\left\{1-\frac{2 J_{1}(j \lambda R)}{j \lambda R J_{0}(j \lambda R)}\right\} e^{j \omega t} .
$$

Substituting Eq. (16) into Eq. (11), and integrating with respect to $r$ from 0 to $R$, we obtain

$$
\left.\begin{array}{r}
-\pi R^{2} \Delta \tilde{p}_{i}\left\{1-\frac{2}{j \lambda R} \frac{J_{1}(j \lambda R)}{J_{0}(j \lambda R)}\right\} e^{j \omega t}=2 \pi \mu_{b} \frac{\Delta \tilde{p}_{i}}{j \omega \rho_{0}} \frac{\lambda R J_{1}(j \lambda R)}{j J_{0}(j \lambda R)} e^{j \omega t} \\
-\pi R^{2} \Delta \tilde{p}_{i} e^{j \omega t}
\end{array}\right\} .
$$

Using the cross-sectional average velocity $\bar{u}$, Eq. (18) becomes

$$
\left.\begin{array}{l}
\rho_{0} A l \dot{\bar{u}}=-2 A \mu_{b} l \Gamma(R) \bar{u}-A \Delta \tilde{p}_{i} e^{j \omega t} \\
\Gamma(R)=\frac{\lambda^{2} J_{1}(j \lambda R)}{j \lambda R J_{0}(j \lambda R)-2 J_{1}(j \lambda R)}
\end{array}\right\},
$$

where $" \cdot "=d / d t$. Equation (19) is the equation of motion of the total shaded area in Fig. 2. On the right-hand side of the first equation of Eq. (19), the first term describes the shear stress from the wall, and there is a phase difference between this term and $\bar{u}$ because $\Gamma(R)$ is a complex number. Therefore, the shear stress from the wall has an effect other than viscous damping.

Now we model the fluid in the shaded area in Fig. 2 as the mass point having base support damping, as shown in Fig. 3 . We relate the average velocity $\bar{u}$ to velocity $\dot{x}_{i}$ at nodal point $i$. Moreover, we assume

$$
\bar{u}=\dot{x}_{i}=\bar{U}_{i} e^{j \omega t} .
$$

Then Eq. (19) becomes

$$
\left\{j \omega \rho_{0} A l+2 \mu_{b} A l \Gamma(R)\right\} \bar{U}_{i}=-A \Delta \tilde{p}_{i} .
$$

On the other hand, the equation of motion of the equivalent concentrated model in Fig. 3 is given by

$$
m \ddot{x}_{i}=-c^{b} \dot{x}_{i}-A \Delta \tilde{p}_{i} e^{j \omega t} .
$$

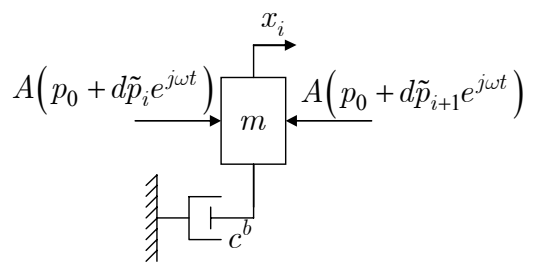

Fig. 3 Mass and base support damper 
Substituting $\dot{x}_{i}=\bar{U}_{i} e^{j \omega t}$ into Eq. (22), we obtain

$$
\left(j \omega m+c^{b}\right) \bar{U}_{i}=-A \Delta \tilde{p}_{i} .
$$

Comparing Eq. (23) and Eq. (21), mass $m$ and $c^{b}$ of the equivalent concentrated model are written as follows:

$$
\begin{aligned}
& m=\rho_{0} A l+2 \mu_{b} A l \frac{1}{\omega} \operatorname{Im}[\Gamma(R)], \\
& c^{b}=2 \mu_{b} A l \operatorname{Re}[\Gamma(R)] .
\end{aligned}
$$

Let $f_{i}^{b}$ be the damping force acting on nodal point $i$ from the base support damper. Then, $f_{i}^{b}$ becomes

$$
f_{i}^{b}=-c^{b} \dot{x}_{i} .
$$

$\operatorname{Re}[\Gamma(R)]$ and $\operatorname{Im}[\Gamma(R)]$ in Eqs. (24), (25) are positive real numbers. Therefore, the equivalent mass $m$ is the sum of $\rho_{0} A l$, which is the mass of fluid in the shaded area, and the apparent mass $2 \mu_{b} A l \operatorname{Im}[\Gamma(R)] / \omega$. The apparent mass describes the coordinate phase component with $\dot{\bar{u}}$ in the shear stress from the wall, and $c^{b}$ describes the coordinate phase component with $\bar{u}$. Also, because $\Gamma(R)$ is a function of $\lambda$ from Eq. (19), $m$ and $c^{b}$ are functions of $\omega$.

Figure 4 shows the velocity distribution in the case the fluid is air. The graphs show the magnitude and the argument of $1-J_{0}(j \lambda r) / J_{0}(j \lambda R)$ in Eq. (16), respectively, when the diameter is $5 \mathrm{~mm}$ and the frequency is $5 \mathrm{~Hz}, 10 \mathrm{~Hz}$, and $20 \mathrm{~Hz}$. As shown in this figure, the shape of $1-J_{0}(j \lambda r) / J_{0}(j \lambda R)$ changes depending on the frequency for both the magnitude and the argument. Therefore, the fluctuation of the velocity distribution with time also varies greatly according to the frequency. However, by using $m$ and $c^{b}$, we can consider the influence of the shear stress from the wall depending on the velocity distribution.

In addition, if we assume the Hagen-Poiseuille flow, the velocity distribution is a quadratic function, and we derive $m$ and $c^{b}$ in a similar way

$$
\begin{aligned}
m & =\rho_{0} A l, \\
c^{b} & =8 \pi \mu_{b} l .
\end{aligned}
$$

As above, in the case of the Hagen-Poiseuille flow, the shear stress from the wall has the effect of viscous damping, but does not have the effect of the mass.

\subsection{Connecting damper}

Let $\tilde{u}$ designate the velocity in the case the radial velocity distribution is uniform, and $\mu_{c}$ is the coefficient of viscosity about the normal stress of fluid. If we use Stokes' assumption, the normal stress acting on the cross section is given by ${ }^{(10)}$

$$
\tau_{x x}=\frac{4}{3} \mu_{c} \frac{\partial \tilde{u}}{\partial x} .
$$

We model the effect of the normal stress as the connecting damper between the nodal

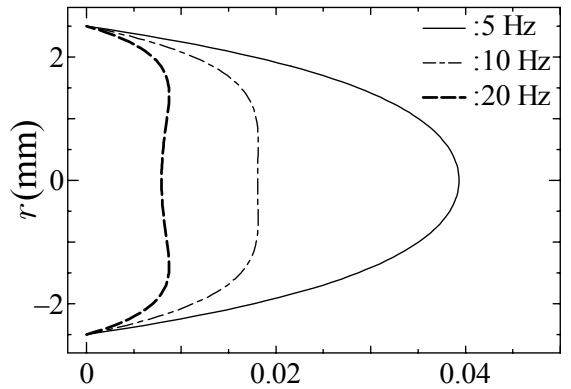

(a) $\left|1-J_{0}(j \lambda r) / J_{0}(j \lambda R)\right|$

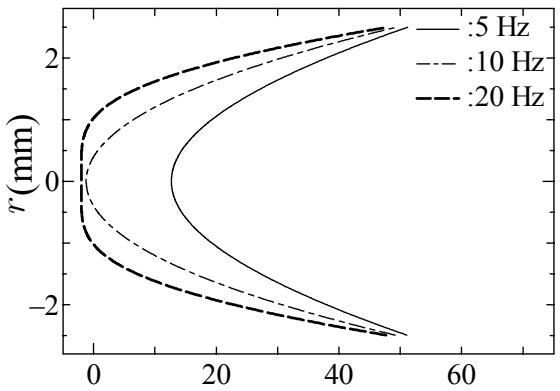

(b) $\arg \left[1-J_{0}(j \lambda r) / J_{0}(j \lambda R)\right](\mathrm{deg})$

Fig. 4 Velocity distribution 
points, as shown in Fig. 1. For that purpose, if we relate velocity $\tilde{u}$ to velocity $\dot{x}_{i}$ and transform to the equation using the finite difference form, we get

$$
f_{i}^{c}=\frac{4 \mu_{c} A}{3 l}\left(\dot{x}_{i}-\dot{x}_{i-1}\right),
$$

where $f_{i}^{c}$ is the damping force from the connecting damper. Therefore, the connecting damping coefficient $c^{c}$ becomes

$$
c^{c}=\frac{4 \mu_{c} A}{3 l} .
$$

\subsection{Equation of motion}

Finally, the equation of motion of the mass point $i$ is written as follows

$$
m_{i} \ddot{x}_{i}=f_{i+1}^{k}+f_{i+1}^{c}-f_{i}^{k}-f_{i}^{c}-f_{i}^{b} .
$$

The nonlinearity of this model is derived from the nonlinear restoring force from the equation of adiabatic change. When we model the fluid as the concentrated mass model, we can solve the problem more easily than by analyzing it by a difference method, because the equation of motion is an ordinary differential equation, as in Eq. (32).

\section{Application to the hydraulic oil tube problem}

\subsection{Analysis object}

To confirm the validity of the equivalent mass and base support damper, derived from the shear stress from the wall in Section 2, we apply them to the wave phenomena in the

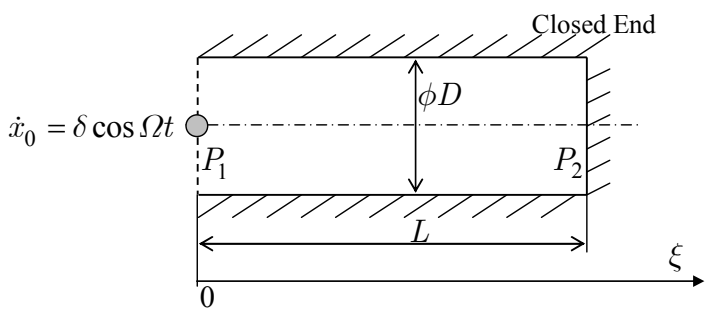

Fig. 5 Model of hydraulic oil pipe

Table 1: Parameter values

\begin{tabular}{c|c||c|c}
\hline \hline$L(\mathrm{~m})$ & 4.18 & $D(\mathrm{~mm})$ & 6 \\
\hline$K(\mathrm{GPa})$ & 1.96 & $\rho_{0}\left(\mathrm{~kg} / \mathrm{m}^{3}\right)$ & 1000 \\
\hline$\mu(\mathrm{Pa} \cdot \mathrm{s})$ & 0.1745 & $\mu_{c}(\mathrm{~Pa} \cdot \mathrm{s})$ & 0 \\
\hline
\end{tabular}

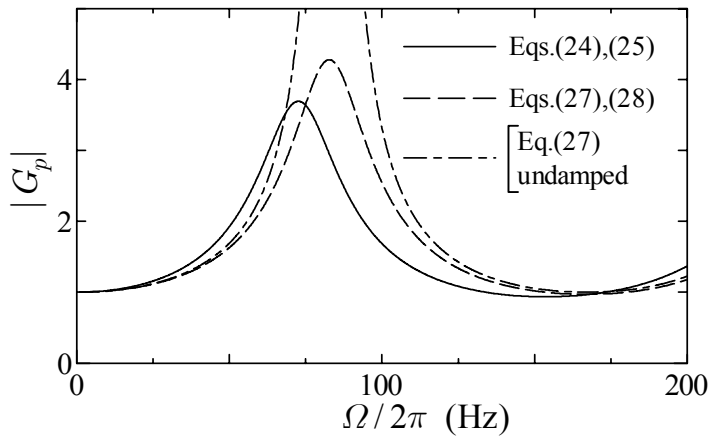

Fig. 6 Numerical computational results of $\left|G_{p}\right|$ 
hydraulic oil tube so that the effect of the viscosity is large. We compare this result with the experimental and numerical result by Okamura et al. ${ }^{(9)}$, who represented the oil pressure wave phenomena in a tube with a uniform cross-section, as shown in Fig. 5, in which the left end is a velocity boundary and the right end is fixed. Letting $P_{1}$ designate the complex amplitude of the pressure at the left end, $P_{2}$ the complex amplitude of the pressure at the fixed end, they obtained by experiment and computation the transfer function defined by the following formula.

$$
\left|G_{p}(j \Omega)\right|=\left|\frac{P_{2}(j \Omega)}{P_{1}(j \Omega)}\right| .
$$

However, they modeled the oil as a continuum and assumed the velocity distribution to be similar to that in Section 2. Moreover, they regarded the fluid as a linear system and considered the effect of the shear stress from the wall, but ignored the damping derived from the normal stress.

We deal with Okamura's problem, and calculate $\left|G_{p}(j \Omega)\right|$ by the transfer matrix method from the concentrated mass model, using the linear constant of a spring in Eq. (8). In this regard, we consider only the base support damping. The partition number $N$ of the oil in the tube is 16 . Table 1 shows the parameter values that satisfy the same condition set by Okamura. The velocity boundary at the left end and the fixed end at the right end are given by

$$
\begin{aligned}
& \dot{x}_{0}=\delta \cos \Omega t, \\
& x_{N}=0,
\end{aligned}
$$

where $\Omega$ is the exciting angular frequency.

\subsection{Numerical results}

Figure 6 shows the numerical results obtained by the concentrated mass model. The solid line shows the result by Eqs. (24) and (25), the dashed line shows the result by Eqs. (27) and (28), the dashed-dotted line shows the result when the base support damper is zero (i.e., the shear stress is neglected). All three results agree well with the numerical results computed by Okamura. Further, these results agree well with the experimental results, so the mass in Eq. (24) and the base support damper in Eq. (25) are valid.

In addition, the peak frequency for the mass in Eq. (27) and the base support damper in Eq. (25) show close agreement with the peak frequency of the dashed line result. Therefore, the base support damper cannot explain the phenomenon that a resonance frequency decreases, so the effect of the mass based on the shear stress from the wall is large.

\section{Application to the sound tube problem}

\subsection{Analysis object}

To confirm the validity of the concentrated mass model proposed in Section 2, we investigate the nonlinear wave phenomena generated in a sound tube, as shown in Fig. 7. The right end of the sound tube is closed. At the left end, the air is excited by the piston, based on the displacement described by the following formula:

$$
x_{0}=\delta \sin \Omega t
$$

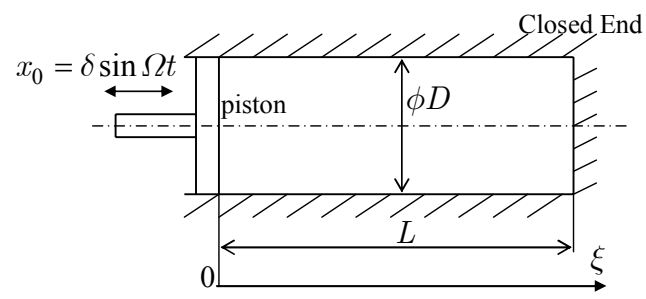

Fig. 7 Model of sound tube 
In a sound tube such as this, a standing wave in a sinusoidal wave configuration is generated if the amplitude of $\delta$ is small, but a shock wave appears at near-resonant frequencies if the amplitude of $\delta$ is large due to the nonlinear effect. An experiment on this phenomena was carried out by Saenger et al. ${ }^{(4),(5)}$. Table 2 shows the parameters of the Saenger's experiment. In this paper, we simulate the pressure wave phenomena with the concentrated mass model, using the nonlinear restoring force in Eq. (5), and the parameters listed in Table 2. In this regard, the viscosity $\mu_{c}$ about the connecting damper is larger than the real value of air. We discuss this point in Section 4.3.

\subsection{Numerical results}

As stated, we simulate the sound tube problem using the concentrated mass model, and obtain a steady periodical solution by a shooting method. The method of the numerical integral is the Runge-Kutta method, the time interval is the subdivision of one cycle into one-thousand-twenty-fourth. The number of partitions $N$ is 64 .

Figure 8 is a comparison of the numerical results by the concentrated mass model and the experimental results by Saenger ${ }^{(5)}$. The left figures show the numerical results and the right figures show the experimental results. These figures describe the time series waveform of $d p$ at the closed end. The scale of the vertical axis of the numerical results and the scale of the experimental results coincide. In addition, all steady periodical solutions by the shooting method in Fig. 8 are stable. As seen in Fig. 8, the numerical results agree well with the experimental results qualitatively and quantitatively in each frequency, except that the amplitude of the numerical result at $104.7 \mathrm{~Hz}$ is smaller than the experimental result. In particular, the shock wave is generated in the experimental result at $100.7 \mathrm{~Hz}$, which is the resonance frequency; this phenomenon is simulated by the concentrated mass model using the nonlinear restoring force in Eq. (5). Moreover, the total amplitude of the pressure of the experimental result at $100.7 \mathrm{~Hz}$ is $28.8 \mathrm{kPa}$, and the total amplitude of the numerical result is $28.9 \mathrm{kPa}$, so both correspond quantitatively. In addition, we cannot simulate a shock

Table 2 Parameter values

\begin{tabular}{c|c|c|c}
\hline \hline$L(\mathrm{~m})$ & 1.7 & $D(\mathrm{~mm})$ & 48 \\
\hline$p_{0}(\mathrm{MPa})$ & 0.1078 & $\gamma$ & 1.4 \\
\hline$\delta(\mathrm{mm})$ & 3.175 & $\rho_{0}\left(\mathrm{~kg} / \mathrm{m}^{3}\right)$ & 1.29 \\
\hline$\mu_{b}(\mathrm{~Pa} \cdot \mathrm{s})$ & $1.82 \times 10^{-5}$ & $\mu_{c}(\mathrm{~Pa} \cdot \mathrm{s})$ & 1.5 \\
\hline
\end{tabular}
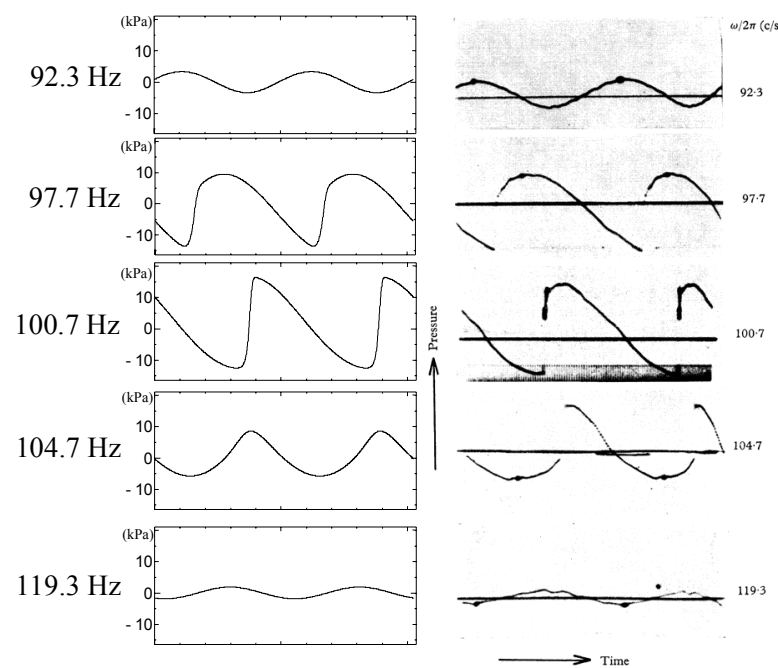

Fig. 8 Numerical and experimental results ${ }^{(5)}$ of pressure wave 
wave like this in the case of the linear restoring force in Eq. (6).

Figure 9 shows the phenomenon that the shock wave generated at $100.7 \mathrm{~Hz}$ propagates through the sound tube. This figure describes the spatial distribution of the pressure at the time, as in the following equation:

$$
t=t_{0}+\frac{2 n \pi}{16 \Omega} \quad(n=1,3,5, \cdots, 15),
$$

where $t_{0}$ is the time when the displacement of the piston is $-\delta$ and the velocity is zero. In the left figure, the shock wave propagates to the right while the piston moves to the right. In the same way, in the right figure, the shock wave propagates to the opposite side while the piston moves to the left. It is common knowledge that the shock wave moves between the piston and closed end ${ }^{(4),(5)}$. Figure 9 is confirmation of this knowledge.

\subsection{Characteristic of the connecting damper}

When we calculate the pressure wave in Fig. 8 and Fig. 9, we assume the value of $\mu_{c}$ is $1.5 \mathrm{~Pa} \cdot \mathrm{s}$ which is larger than the real value of air $\left(1.82 \times 10^{-5} \mathrm{~Pa} \cdot \mathrm{s}\right)$. If we make the value of $\mu_{c}$ smaller, a numerical high-frequency vibration is generated after the pressure wave rises straight up, as shown in Fig. 10. Figure 10 shows the time-series pressure waveform at the closed end at $100.7 \mathrm{~Hz}$ when $\mu_{c}$ changes.

Because the velocity greatly changes in the shock wave with a thickness of several micrometers, the value of $\partial \tilde{u} / \partial x$ in Eq. (29) is large. In contrast, because the length of elements of the concentrated mass model is longer than the thickness of the shock wave, $\left(\dot{x}_{i}-\dot{x}_{i-1}\right) / l$ in Eq. (30) transformed from $\partial \tilde{u} / \partial x$ using the finite difference form is

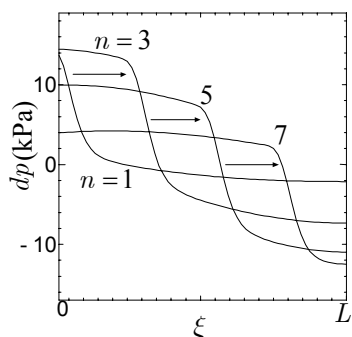

$n=1,3,5,7$

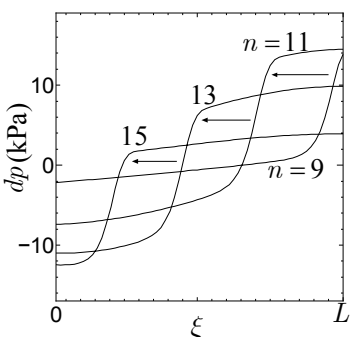

$n=9,11,13,15$

Fig. 9 Pressure wave propagation $(\Omega / 2 \pi=100.7 \mathrm{~Hz})$
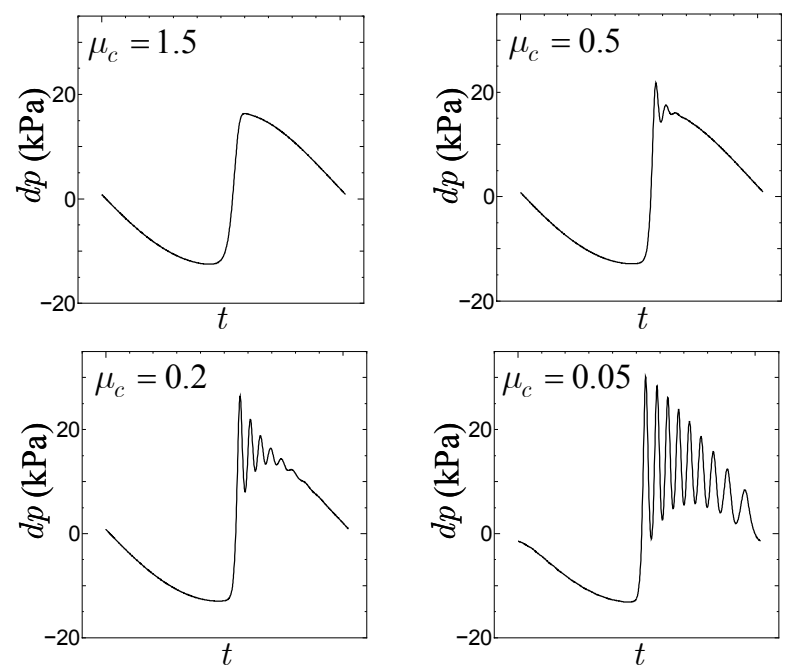

Fig. 10 Time history of pressure wave $(\Omega / 2 \pi=100.7 \mathrm{~Hz})$ 
smaller, and so the connecting damping force $f_{i}^{c}$ is smaller than the real value. Therefore, numerical vibration appears. This discussion is valid because the value of $\mu_{c}$ that prevents numerical vibration decreases as the partition number $N$ increases, and numerical vibration is not generated even if $\mu_{c}$ is the real value of air, in the case that the shock wave does not appear.

In our method, in order to handle the numerical vibration problem, we make $\mu_{c}$ larger until the numerical vibration does not appear. Even if we make $\mu_{c}$ larger by this method, the numerical results agree well with the experimental results. The reason is the effect of the connecting damper to all systems is small because $\partial \tilde{u} / \partial x$ is not large in the outer region of the shock wave.

In addition, the appearance of a similar phenomenon is known in the case that the shock wave is analyzed by the finite difference method. In this method, the generation of numerical vibration is suppressed by a dissipation term called artificial viscosity ${ }^{(11)}$.

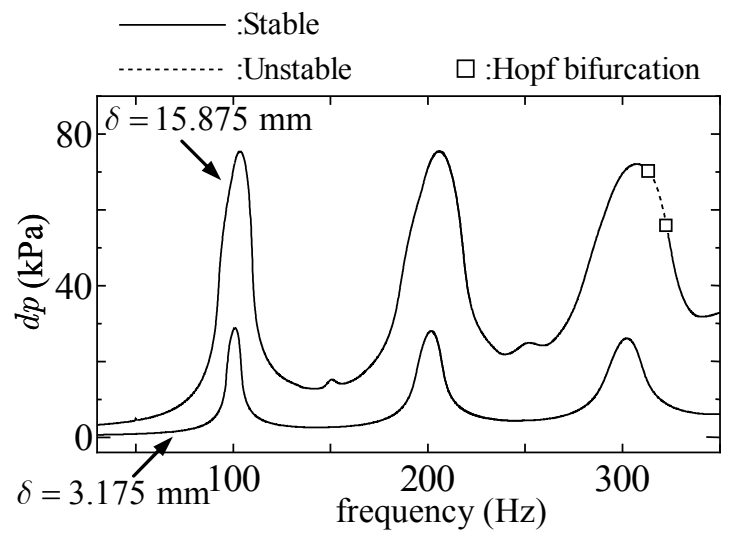

Fig. 11 Frequency response

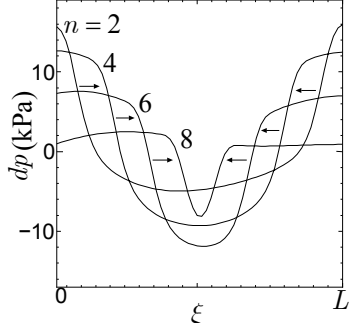

$r=2,4,6,8$

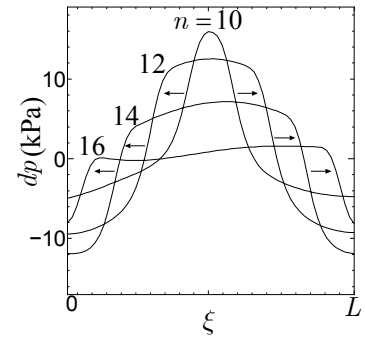

$r=10,12,14,16$

Fig. 12 Pressure wave propagation $(\Omega / 2 \pi=201.7 \mathrm{~Hz})$

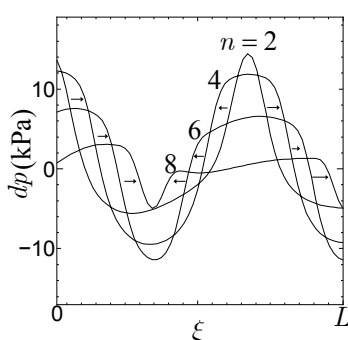

$r=2,4,6,8$

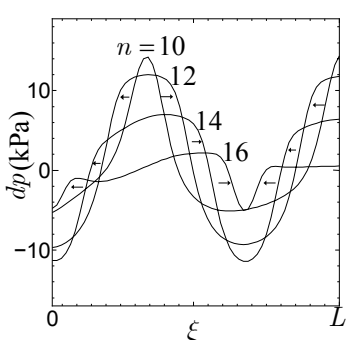

$r=10,12,14,16$

Fig. 13 Pressure wave propagation $(\Omega / 2 \pi=302.2 \mathrm{~Hz})$ 


\subsection{Frequency response}

Figure 11 shows the frequency response with the increasing frequency of the piston. The vertical axis shows the total amplitude of the pressure at the closed end. Two curved line are the numerical results when the amplitude of piston $\delta$ is $3.175 \mathrm{~mm}$ and the coefficient of viscosity $\mu_{c}$ is $1.5 \mathrm{~Pa} \cdot \mathrm{s}$, and when $\delta=15.875 \mathrm{~mm}$ and $\mu_{c}=2.0 \mathrm{~Pa} \cdot \mathrm{s}$. The parameters other than $\delta$ and $\mu_{c}$ are used as the values in Table 2. The solid line is the stable solution, the dashed line is the unstable solution, and $\Upsilon$ is a Hopf bifurcation point.

From Fig. 11, there are secondary resonances near $200 \mathrm{~Hz}$, and third-order resonances near $300 \mathrm{~Hz}$. Figures 12 and 13 show the conditions that the shock waves propagate at these resonance points. These figures are similar to Fig. 9. At the secondary resonance, two shock waves travel forward and backward in the sound tube. At the third-order resonance, three shock waves travel in the same manner. In addition, when the amplitude of the piston is $15.875 \mathrm{~mm}$, peaks appear near $150 \mathrm{~Hz}$ and near $250 \mathrm{~Hz}$. These peaks describe the secondary higher harmonic resonances of the third mode and fifth mode. Further, between $313.6 \mathrm{~Hz}$ and $322.6 \mathrm{~Hz}$, there is the unstable solution from the Hopf bifurcation, and a quasi-periodic solution is generated in this frequency region.

As shown above, we can easily obtain a detailed frequency response by use of the shooting method applying the concentrated mass model.

\section{Application to the progressive wave problem}

A progressive wave that is generated by a train in the tunnel also changes to a shock wave. Kamakura ${ }^{(7)}$ carried out the experiment to investigate this phenomenon. He observed the formation of a shock wave generated by a horn driver in a straight tube. In this section, we analyze this experiment, and confirm the validity of the concentrated mass model for the progressive wave problem.

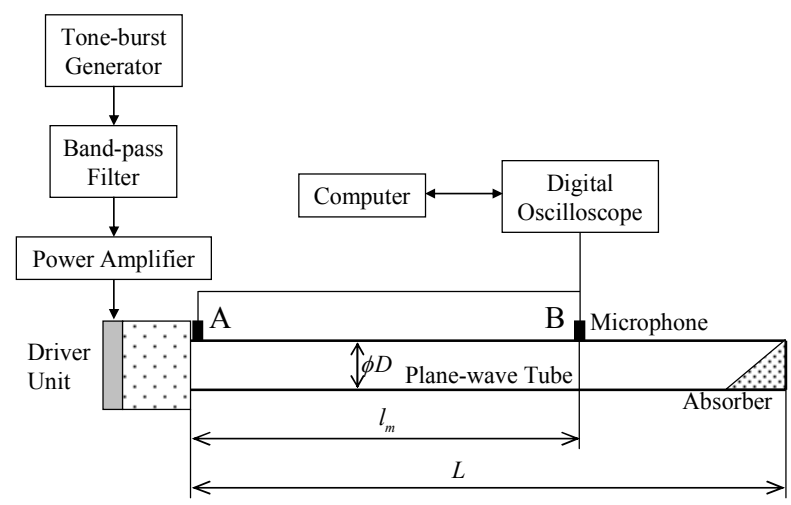

Fig. 14 Experimental apparatus

Table 3: Parameter values

\begin{tabular}{c|c|c|c}
\hline \hline$L(\mathrm{~m})$ & 20 & $l_{m}(\mathrm{~m})$ & 17.5 \\
\hline$D(\mathrm{~mm})$ & 49 & $p_{0}(\mathrm{MPa})$ & 0.1078 \\
\hline$\gamma$ & 1.4 & $\rho_{0}\left(\mathrm{~kg} / \mathrm{m}^{3}\right)$ & 1.29 \\
\hline$\mu_{b}(\mathrm{~Pa} \cdot \mathrm{s})$ & $1.82 \times 10^{-5}$ & $\mu_{c}(\mathrm{~Pa} \cdot \mathrm{s})$ & 0.04 \\
\hline$\Delta p_{0}(\mathrm{~Pa})$ & $1285,128.5$ & $N$ & 8000 \\
\hline
\end{tabular}




\subsection{Experimental apparatus and condition of numerical calculation}

Figure 14 shows the experimental apparatus used by Kamakura. The horn driver is attached to the left side of the tube. Glass fiber is placed at the right end of the tube to suppress the generation of a reflected wave. A $1 \mathrm{kHz}$ sinusoidal wave generated by the horn driver is adjusted so that the pressure amplitude $\Delta p_{0}$ at the inlet point becomes $1285 \mathrm{~Pa}$ or $128.5 \mathrm{~Pa}$. At the inlet point (point A) and the point at a distance of $17.5 \mathrm{~m}$ from the inlet point (point B), the pressure wave is measured by microphones. Table 3 shows the parameters of this experiment. We use these parameters rather than the viscous modulus of connecting damping $\mu_{c}$. For $\mu_{c}$, we set the value at $0.04 \mathrm{~Pa} \cdot \mathrm{s}$, which is larger than the real value of air. The boundary condition at the inlet point is the pressure boundary condition. The force $f_{0}$ acting on the nodal point 0 from the left side is given by

$$
f_{0}=\Delta p_{0} A \sin \Omega t .
$$

The partition number $N$ of the concentrated mass model is 8000 , the time step size is $10^{-5} \mathrm{~s}$, and the numerical integration method is the Runge-Kutta method. In this problem, the partition size and time step size must be small because the frequency of the excitation force is high $(1 \mathrm{kHz})$. However, because the partition size is small, the value of $\mu_{c}$ which suppresses the generation of the numerical vibration is smaller than that in the sound tube problem.

\subsection{Numerical results}

Figure 15 shows the numerical result and Figure 16 shows the experimental result by Kamakura $^{(7)}$. The left figures show the time-series pressure wave at point $\mathrm{A}$ and the right figure show the wave at point B (7000th element). The solid lines show the result when the amplitude of pressure at entrance $\Delta p_{0}=1285 \mathrm{~Pa}$, the dashed lines show the result when $\Delta p_{0}=128.5 \mathrm{~Pa}$. The value of the dashed line is ten times as large as the real value. The positive peak value and the negative peak value of the numerical results at point $\mathrm{B}$ in the case of $\Delta p_{0}=1285 \mathrm{~Pa}$ are different, but we eliminate the offset in Fig. 15 in order to fit the indicated means in Fig. 16. The initial condition of the numerical calculation is $d p_{i}=0, x_{i}=0, \dot{x}_{i}=0$, and the right end is the fixed end. We remove the first wave in which the effect of a transient response exists, and adopt the result before the reflected wave arrives at point $\mathrm{B}$.

The numerical result at point $\mathrm{B}$ agrees well with the experimental result qualitatively and quantitatively in the case of $1285 \mathrm{~Pa}$ (solid line) and $128.5 \mathrm{~Pa}$ (dashed line). Especially

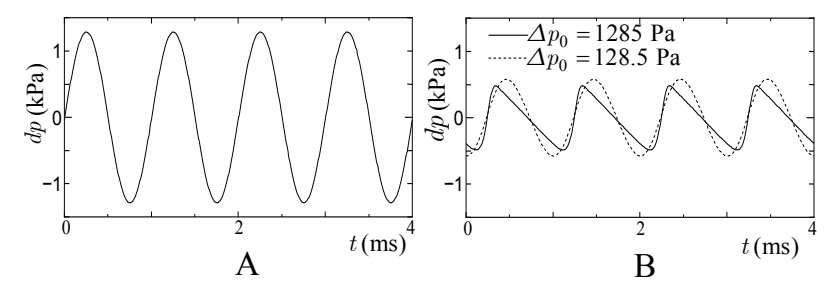

Fig. 15 Numerical result of pressure wave

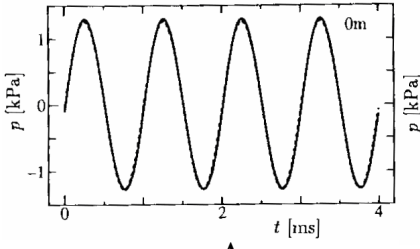

A

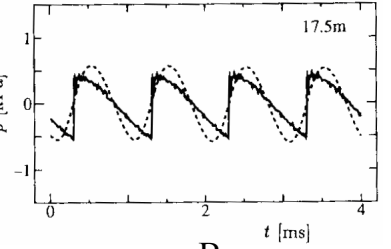

B

Fig. 16 Experimental result of pressure wave ${ }^{(7)}$ 
in the case of $1285 \mathrm{~Pa}$, the shock wave that stands straight up appears at point $\mathrm{B}$; in the case of $128.5 \mathrm{~Pa}$, the shock wave does not occur because the amplitude is small. Comparing the waveform at point $\mathrm{A}$ with that at point $\mathrm{B}$, the numerical result and experimental result at point $\mathrm{B}$ both decrease. Therefore, the phenomenon that a progressive wave attenuates is accurately simulated by the base support damping based on the shear stress from the wall.

As shown above, the concentrated mass model proposed in this paper is valid for the progressive wave problem.

\section{Conclusion}

To analyze the one-dimensional nonlinear pressure wave phenomenon in a cylindrical tube, we propose the concentrated mass model that consists of the equivalent mass, equivalent base support damper, nonlinear spring, and connecting damper. The equivalent mass and damping coefficient of the base support damper are derived from a viscosity and velocity distribution, the nonlinear spring characteristic is derived from the adiabatic change of fluid, and the connecting damper is derived from a normal stress of the viscosity. Furthermore, we confirm the validity of the concentrated mass model by comparing the numerical results computed by this model with some typical experimental results. Especially for the nonlinear pressure wave problem in the sound tube problem, we can obtain results that agree well with the experimental results calculated by Saenger ${ }^{(5)}$ qualitatively and quantitatively, such as the phenomenon that a shock wave occurs near resonance frequencies. In addition, regarding the phenomenon that a progressive wave changes to a shock wave, we can also obtain results that agree well with the experimental result calculated by Kamakura ${ }^{(7)}$. In addition, we clarify that adjusting the coefficient of viscosity of the connecting damper is effective for the numerical vibration problem specific to a shock wave analysis.

In summary, the concentrated mass model we propose in this paper can obtain high-accuracy numerical results even though it is a simplified model. This model is valid for the numerical analysis of nonlinear pressure wave problem.

\section{Acknowledgment}

This work was supported by Grant-in-Aid for JSPS Fellows (No. 21-2156).

\section{References}

(1) Okada, M. et al., Analysis of Generation Mechanism for Abnormal Noise Emitted from Exhaust Outlet, Transactions of the Society of Automotive Engineers of Japan, Vol. 32, (1986), pp. 79-85.

(2) Sekine, N. et al., Study of Silencer Characteristics in a Gas Flow with Shock Wave, Transactions of the Japan Society of Mechanical Engineers, Series B, Vol. 60, No. 575 (1994), pp. 2315- 2320.

(3) Mashimo, S. et al., Attenuation and Distortion of a Compression Wave Propagation in a High-Speed Railway Tunnel, Transactions of the Japan Society of Mechanical Engineers, Series B, Vol. 62, No. 597 (1996), pp. 1847- 1854.

(4) Saenger, R. A., et al., Periodic Shock Waves in Resonating Gas Columns, Journal of the Acoustic Society of America, Vol. 32, No. 8 (1960), pp. 961- 970.

(5) Chester, W., Resonant oscillations in closed tubes, Journal of Fluid Mechanics, Vol. 18 (1964), pp. 44- 66.

(6) Arakawa, M. et al., Nonlinear Phenomena Induced by Finite-Amplitude Oscillation of Air Column in Closed Tube, Transactions of the Japan Society of Mechanical Engineers, 
Series B, Vol. 60, No. 580 (1994), pp. 4052- 4058.

(7) Kamakura, T., Fundamentals of Nonlinear Acoustics, (1996), p. 216, Aichi-shuppan.

(8) D'Souza, A. F., et al., Dynamic Response of Fluid Lines, Transactions of the ASME, Ser.D, Vol. 86, No. 3 (1964), pp. 589- 598.

(9) Okamura, K. et al., Research on Propagation Phenomena in Hydraulic Line, Transactions of the Japan Society of Mechanical Engineers, Part 2, Vol. 38, No. 314 (1972), pp. 26122620.

(10) Takori, T., Fluid Engineering, (1989), p. 28, University of Tokyo Press.

(11) Roache, P. J., Computational Fluid Dynamics, (1976), p. 351, Hermosa Publishers. 